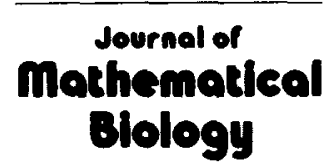

(C) Springer-Verlag 1994

\title{
Simple models for trail-following behaviour; Trunk trails versus individual foragers
}

\author{
Leah Edelstein-Keshet \\ Mathematics Department, University of British Columbia, Vancouver, B.C. V6T 1Z2, Canada
}

Received 7 October 1992; received in revised form 28 April 1993

\begin{abstract}
Social insects such as ants use trial-marking and trail-following to organize the behaviour and movement patterns of a large population. Since behaviour has to meet needs of the population in a changing environment, the type of trail networks formed must be adaptable. Both solitary foraging as well as mass migration along a system of trunk trails are behaviours essential for survival of the colony, and the population must be able to switch from one behaviour to the other, depending on conditions. Using a mathematical model for trail following we show that subtle changes in individual behaviour can give rise to dramatic differences in the behaviour of the population, including the ability to switch from solitary movement to organized group traffic. The model incorporates biological parameters associated with the organism, the trail-marker, and the population. Ordinary differential equations are formulated for the density of the trails and for the number of individuals following trails or exploring randomly. It is assumed that the followers reinforce trails by pheromone marking, and that individuals respond to the strength of the trails by becoming more efficient followers. The model is analyzed by qualitative phase-plane methods.
\end{abstract}

Key words: Trail-following - Ant trail pheromone - Self-organization - Collective behaviour - Low vs high traffic networks

\section{Introduction}

By following a chemical trail deposited by their sisters, ants are able to move in a coordinated way as a group. The chemical marker, called a pheromone is a volatile substance painted on the path of the moving ant. As the substance diffuses into the air, it creates a corridor of scent to which other ants will respond and orient (Bossert and Wilson 1963). Receptors on the two antennae of an ant can sense pheromone, and by a comparison of left and right levels, prompt the ant to turn onto a trail it encounters. There is a keen but finite tolerance in the pheromone receptors, and 
errors such as losing a trail, or turning towards the direction of decreasing pheromone occur.

At the level of the society, coordinated response to a threat by predators, collective effort to feed the colony, and organized migratory movements are essential to survival. Yet, the coordination of the movements of thousands of individuals is not governed by leaders or central decision makers. Rather, the organized behaviour is the outcome of a collective behaviour that has been called "self-organization" (Camazine 1991, Aron et al. 1989, 1990; Beckers et al. 1989, 1990; Calenbuhr and Deneubourg 1989, 1990; Deneubourg et al. 1989, 1990; Pasteels et al. 1987a, b, c and numerous references therein.) The model in this paper explores how selforganized systems of trails can change and respond to changes in the needs of the society or the environmental influences it faces: for example, at times, a diffuse network of trails serves to efficiently cover a wide area over which the population searches for food. At other times, mass migration to relocate to a new nest, or tight columns to exploit a recently discovered food source are required, see Fig. 1. (See, for example, Hölldobler and Wilson 1990, and references therein.) The way that the population adapts to these extremes, and the way this is achieved by slight alterations in individual responses are the subject of this paper.

The main thesis of this paper is that properties and behaviours at the level of the individual, together with appropriate interactions between the individuals and their trails can account for the plasticity of trail networks and their function. The specific properties of individuals that enter into the model are the rates of secretion and evaporation of pheromone or other marker, the fidelity of followers (i.e., their probability of tracking the path per unit time or distance), and the attraction of the trails towards other individuals. (These parameters are, in principle, experimentally measurable; some values are obtainable from the literature.) The model characterizes the complex spatio-temporal evolution of trail networks by a gross attribute, the density of traffic (ratio of followers to trail length). Other work on this subject (e.g. Watmough 1992) deals with a fuller spatio-temporal model.

The paper is organized as follows: Section 1 contains background motivation. In Sect. 2 the model is derived, and assumptions are explained and justified in detail. In Sect. 3 the behaviour of the model is analyzed. Implications and a discussion follow in Sects. 4 and 5.

\section{Motivation}

While this paper is concerned with a mathematical model, the research on this problem began with a set of simulations written in C by Bard Ermentrout, and modified from cellular automata depicting motion of cells. (Edelstein-Keshet and Ermentrout 1990, Ermentrout and Edelstein-Keshet 1993). Positions of individual "ants" and the strength of their trails are represented by pixels, and simple rules governing the motion and the interaction of individuals with the trails postulated. Initially, "ants" are "released" randomly in an arena, and allowed to move in arbitrary initial directions. The parameters governing their motion, the strength of their interaction, etc. can be varied, and the resulting animated population swarming is observed. In recent simulations written by James Watmough (now a graduate student with the author at UBC), ants are "released" from a single point, "the nest" and allowed to wander over a rectangular area with periodic boundary conditions. It is thus possible to 
follow the spatial organization both in a totally random initial configuration, and in one in which all individuals start at the same point. We report on these simulations in a separate paper.

By experimenting with these simulations, it becomes evident that slight changes in initial conditions, or slight changes in certain key parameters result in different possible outcomes: Either (a) the network of trails, composed mainly of weak, shortlasting paths changes continuously, with no order or persistent structure, or (b) a few relatively stable and strong trails emerge, with most of the traffic exclusively along these trails. However, it is difficult to understand exactly how the changes in group behaviour depend on parameters from simulations alone. This motivated our trying to understand the phenomenon by considering a mathematical model.

Natural ant colonies also display fascinating trail configurations. Sketches of trail networks of ant swarms in Schneirla (1971), Rettenmeyer (1963), Raignier and van Boven (1955), demonstrate variations in the structure and density (see Figure 1). These variations are correlated with the function of the trails. Exploration trails appear to densely cover an area, with fine lace-like networks, whereas trunk trails from a nest to a food source, or migratory routes are much more direct, less frequently branched, and capable of carrying a much greater volume of traffic. The investigation of these naturally occurring trail networks provides a challenge: to try to understand how these form, and what governs adaptation from one type of network to another.

Ultimately, one can pose questions about the way that the behaviour of the social organism has evolved. Since we have already argued that the ability of the population to switch between one type of network and another is an essential adaptation to the resource distribution, the changes in the colony, in the environment, and in the tasks and problems to be solved, it is reasonable to assume that colonies with the ability to adapt their organization to these needs have a selective advantage, and hence should be selected for by evolution. The model we present below should allow us to investigate what features of the individual could have evolved through natural selection to lead to plasticity in the behaviour of the population.

\section{A model for trail-following behaviour}

Many different modelling approaches are possible in describing trail networks and movement patterns of organisms following these. We review some of the preexisting theoretical and experimental work in Sect.5. Briefly, the Brussels group (Deneubourg, Pasteels, Aron, and Goss) have investigated binary choices made by ants among branches of an artificial system of bridges. They have also pioneered simulations showing swarming behaviour. Others (Calenbuhr and Deneubourg) deal with the individual responses to pheromone, or the rate of secretion and evaporation of the active space surrounding a trail (Bossert and Wilson).

In this paper, the aim is to analyze the transitions between trunk trails and solitary foraging. The model is kept purposely simple in an attempt to reveal some of the important mechanisms of the collective behaviour. It is felt that including many fine details of the biology, or treating a complex spatio-temporal model should be attempted only after some initial understanding of simpler models is achieved. We define the following variables and parameters with corresponding dimensions $(\mathrm{d}=$ distance, $\mathrm{A}=$ area, $\mathrm{t}=$ time $)$

$T(t)=$ total length of trails per unit area at time $t$

$\left[\mathrm{dA}^{-1}\right]$, 


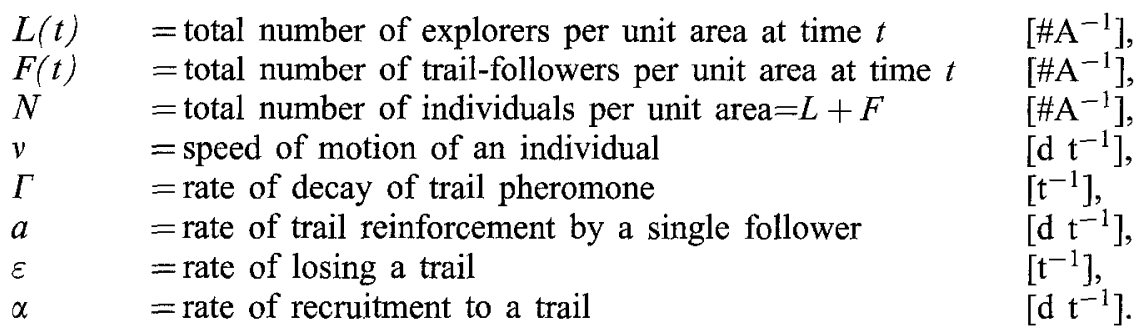

It is advantageous to define $T(t)$ as length-density, rather than number-density of trails. Trail networks contain many intersecting trails, leading to ambiguity in identifying and counting individual trails. The total length of trails inside a region of unit area can be unambiguously defined and measured (e.g. by digitizing and numerically integrating the length of trail segments). A similar approach has been used by the author in modelling density of branches in a colony of fungi and other branched networks (See Edelstein 1982, Edelstein-Keshet and Ermentrout 1989).

For the purpose of the model we consider only two types of individuals, those who are exploring $(L(t))$ and those following pre-existing trails $(F(t))$. Parameters are assumed to be positive constants. Eventually we will be concerned with variations of the parameters (typical of changes in the environmental conditions, for example). The equations of the model are derived below.

\section{2.a Trail behind a single individual}

Bossert and Wilson (1963) characterized the length of a "scent corridor" behind a single ant. This length is the distance between the point at which the fresh trail is being deposited, and the fade-out point, at which it is no longer perceptible. When the ant first starts to move and deposit a trail, the length of trail will gradually increase until it reaches this finite length, at which the rate of deposition at the leading portion just balances the rate of fading at the rear. For this reason, the length of trail secreted by a single ant will be described by the equation:

$$
\frac{d T}{d t}=v-\Gamma T .
$$

Here $v$ is the length deposited per unit time, equivalent to the walking speed of an ant laying a fresh trail, and $\Gamma T$ is the decay rate per unit time). The two terms in this equation balance when $T=v / \Gamma$. The trail behind a single ant will be called a simple trail, and its length will be represented by the quantity

$$
d_{s}=v / \Gamma=\text { the length of a simple trail . }
$$

Bossert and Wilson (1963) calculated that this length is roughly $28 \mathrm{~cm}$ for the ant Solenopsis saevissima, based on diffusion of the pheromone.

\section{2.b Trails formed by $N$ individuals}

When there are many individuals, we distinguish between those that are making fresh trails, $L(t)$, and those following trails $F(t)$. Both types are assumed to secrete trail-marker, whether to lengthen or to reinforce the trails. The distance associated 
with followers on a trail would in general be shorter than the length of a simple trail, since otherwise the "chain of individuals" on the trail (a term suggested by Calenbuhr 1990) would be broken by fade-out points between them. We allow for the possibility that individuals of type $F$ or $L$ may thus contribute differently to trail length. The equation proposed for the total length of trails due to these is:

$$
\frac{d T}{d t}=v L+a F-\Gamma T
$$

The parameter $a$ has the same dimensions as $v$, i.e, $\left[\mathrm{dt}^{-1}\right]$, and the dimensionless ratio $a / v$ represents the relative contributions to lengthening the trail by followers and explorers. Equation (2) is an approximation which does not incorporate the chemical level of the pheromone on the trails. In the Appendix to this paper we discuss the connection between this phenomenological equation and a material balance of pheromone concentration.

Though Eq.(2) is approximate, it provides a useful characterization of the trail network. In particular, it is consistent with the idea that the trails will have a different total length when the foragers are moving as individuals, versus trails formed through coordinated following. Indeed, from (2) we note that at equilibrium $(d T / d t=0)$, if most individuals are exploring, $(L \sim N$ and $F \sim 0)$, then the total trail density is

$$
T=N v / \Gamma=N d_{s}
$$

However, if most individuals are on trails $(F \sim N, L \sim 0)$ then the total length of the trails is

$$
T=N a / \Gamma=N d_{f}
$$

where we have defined the distance

$$
d_{f}=a / \Gamma=\text { average distance between followers on a trail } .
$$

For a typical example, Wilson (1971) states that 10-20 workers of the ant Camponotus paria follow single file behind one leader, with a distance of separation $5-10 \mathrm{~cm}$. The ratio $\Gamma / a$ represents the mean density of followers per unit length of trail. Further, our previous remark implies that $d_{f}<d_{s}$ since followers are closer to each other than the fade-out distance.

When a trail network is first created, the number of followers and explorers will fluctuate, since some followers will lose the trail whereas some "lost" ants will be attracted to the trail. Mass action kinetics, representing "binding" and "unbinding" to trails are used to model this exchange, leading to:

$$
\begin{gathered}
\frac{d F}{d t}=-\varepsilon F+\alpha L T, \\
\frac{d L}{d t}=\varepsilon F-\alpha L T .
\end{gathered}
$$

The dimensions of the parameter $\alpha$ are Area per unit time per unit distance, $\left[A t^{-1} d^{-1}\right]$, or simply $\left[\mathrm{d} \mathrm{t}^{-1}\right]$. The parameter $\varepsilon$ is the rate that followers lose the 
trail, and has dimensions of $\left[\mathrm{t}^{-1}\right]$. We observe that the quantity $N=L+F=$ total population density is conserved by the above equations. Therefore, we can eliminate one variable, for example $L$, from the equations. The reduced model that results is then:

$$
\begin{gathered}
\frac{d T}{d t}=\nu(N-F)+a F-\Gamma T, \\
\frac{d F}{d t}=-\varepsilon F+\alpha(N-F) T .
\end{gathered}
$$

Equation (5.a, b) can be studied with standard qualitative methods. If all the parameters defined above are constant, there is a single stable steady state, in which a fixed proportion of individuals are following trails. This basic model is not very interesting, and cannot account for any behavioral transitions. However, as we shall see below, a minor but important modification of the model leads to much more interesting and versatile behaviour.

\section{2.c Strength of the trails}

Since ants are a social organism, it is reasonable to expect that the probability of finding and/or staying on the trails would increase as ants are "recruited" by their sisters. Recruitment can take place in different ways. We have already discussed the effect of chemical messages; it is known that the intensity of response to a trail marker depends on chemical strength of the pheromone. When there are many followers reinforcing a system of trails, the level of pheromone marker on the trails increases, so that more individuals are attracted to the trails. However, possibly a second type of recruitment is direct antennal contact between ants; those on a promising route reinforce each other's tendency to stay on the trail, or persuade newcomers to join in. In both types of recruitment, presence of followers enhances the probability that new individuals will be recruited, or that current followers will remain on the trails.

In a desire to keep the model fairly simple, the detailed distribution of pheromone along the trails is not described. Rather, we use a gross indicator of trail strength, the density of traffic, defined as the ratio of followers to a unit length of trail. By the above comments, it is reasonable to suppose that when this ratio is high, the trails become more attractive. (The higher the density of followers along a unit of trail, the greater the number of opportunities for communicating the message to follow the trail.) We loosely define "the trail strength", $S(t)$, by:

$$
\begin{array}{r}
S(t)=\text { strength of the trail network }=F(t) / T(t)=\text { density of followers per unit } \\
\text { length of trail (traffic density). }
\end{array}
$$

The variable $S$ can be used as an indication of the level of coordinated movement versus exploratory movement in the population: Observe that when all individuals are explorers, $L=N, F=0$, so that $S=0$ (We shall call this a weak trail network). When the whole population is following trails, $L=0, F=N, T=a N / \Gamma$ so $S=\Gamma / a$ (This will be a network of maximal strength, and will be called a strong trail network). Note that even though the average distance between followers on a trail, $d_{f}$ is a function of fixed parameters, the above defined variable $S=F / T$, which has dimensions of (1/distance between followers) is not fixed; $S$ is an average 
taken over all trails, including simple fresh trails that have no followers on them, while $d_{f}$ is the average separation distance between followers on a single trail on which they are walking. In the Appendix, we show that under certain assumptions, $S(t)$ is approximately equivalent to the pheromonal strength of the trails, but this is not an essential assumption of the model.

The trail strength $S$ could influence several parameters in the model, such as the rate of reinforcement of a trail by a follower, $a$, the likelihood that a follower loses a trail, $\varepsilon$, as well as the attractivity of a trail to lost ants, $\alpha$. (The other parameters defined in the model are not likely influenced). It is possible to exhaustively deal with each of these possibilities, but many of them are essentially equivalent. We shall explore the consequences of one particular assumption, namely that followers have increased fidelity (decreased drop-off rate, $\varepsilon$ ) when the traffic density is high.

\section{2.d Individuals follow stronger trails more faithfully}

It is reasonable to assume that the stronger the trail, the lower is the likelihood that a follower will lose it. We suppose that the loss rate, $\varepsilon$, is a decreasing function of trail strength, for example

$$
\varepsilon(S)=E \exp (-b S)=E \exp (-b F / T) .
$$

where $E$ is the rate of losing the trail by a follower in the absence of the group recruitment effect (i.e. when the trail is a weak one, $S=0$ ) and $b$ is a parameter that governs how rapidly this rate decreases as the strength of the trail increases.

By using this assumption in Eqs. (5) we obtain a modified model incorporating "trail strength" as follows:

$$
\begin{aligned}
& \frac{d T}{d t}=v(N-F)+a F-\Gamma T, \\
& \frac{d F}{d t}=-E F e^{-b \frac{F}{T}}+\alpha(N-F) T .
\end{aligned}
$$

It is of interest to determine how the length of the trails and the fraction of the population moving along these trails evolves over time from a variety of initial conditions, and under different values of the parameters. Analysis of this model is carried out in the next section.

\section{Analysis of the model}

\section{3.a Reduction to dimensionless form}

To put the equations in dimensionless form we define the dimensionless variables $T^{*}, L^{*}, F^{*}, t^{*}$, as follows:

$$
\begin{aligned}
T & =T^{*} N d_{s}=T^{*} N v / \Gamma \\
L & =L^{*} N \\
F & =F^{*} N \\
t & =t^{*}(1 / \Gamma) \\
S & =F / T=\left(F^{*} / T^{*}\right)(\Gamma / v) .
\end{aligned}
$$


Thus trail length is scaled so that a maximum value of 1 corresponds to the total lengths of simple trails behind $\mathrm{N}$ solitary individuals (the maximal possible length of trails sustained by a population of $\mathrm{N}$ ants), densities of followers and lost individuals are scaled in units of the total population $\mathrm{N}$, and time is scaled in units of the pheromonal half-life $1 / \Gamma$, the time it takes a trail to evaporate to $1 / 2$ its original length. The dimensionless trail strength $S$ would be zero when all the ants are explorers $(F=0)$, whereas it would attain a maximal value of $v / a>1$ when all the ants are following each other at the usual inter-follower distance on the trails. By substituting these expressions into Eqs. $(7 \mathrm{a}, \mathrm{b})$, rearranging, and then dropping the ${ }^{*}$ s, we obtain:

$$
\begin{aligned}
& \frac{d T}{d t}=(1-F)+A^{\prime} F-T, \\
& \frac{d F}{d t}=-E^{\prime} F e^{-B^{\prime} \frac{F}{T}}+\alpha^{\prime}(1-F) T .
\end{aligned}
$$

where the new dimensionless parameters are:

$$
A^{\prime}=\frac{a}{v}, \quad B^{\prime}=\frac{b \Gamma}{v}, \quad E^{\prime}=\frac{E}{\Gamma}, \quad \alpha^{\prime}=\frac{\alpha \nu N}{\Gamma^{2}} .
$$

$A^{\prime}$ is the ratio of length of a simple trail (behind a single ant) to the trail length associated with a follower, and is typically less than 1 , by previous arguments. $B^{\prime}$ is the natural logarithm of the ratio of maximal to actual drop off rates $(E / \varepsilon)$ when the average traffic density is one follower per length $d_{s}$ of the trail (This is an intermediate strength of trails which would occur when part of the population is exploring and part is closely spaced followers). For example, when $B^{\prime}=4$, a follower stays on the trail 55 times longer than it would were it not being recruited to the trail by the other traffic (because $E / \varepsilon=e^{4}=54.6$ ) The dimensionless parameter $E^{\prime}$ is the ratio of trail half life to the half life of a follower on a weak trail, and could take on a wide range of values depending on the type of pheromone, the species of ant and the conditions. Here we assume that trails are more long-lived than the average time spent on the trail by a follower who is not being actively recruited. The parameter $\alpha^{\prime}$ represents the probability that a single ant would be recruited to a trail network whose total density is $N d_{s}$ during the trail half life, and could also vary between extremes.

By arguments given above, we expect that values of the new dimensionless variables in these equations are restricted to the following ranges:

$$
\begin{aligned}
& 0<T<1, \\
& 0<L<1, \\
& 0<F<1, \\
& 0<S<v / a .
\end{aligned}
$$

In Sect. 3.c we analyze the behaviour of Eqs. $(8 \mathrm{a}, \mathrm{b})$. It will be shown that the above model has bi-stable behaviour under a certain set of conditions, and that it can describe behavioral transitions.

To understand what the model predicts, we need to appreciate the qualitative effects of the dimensionless parameters, for example through phase plane analysis. 
One difficulty in the analysis stems from the fact that the locus of points satisfying $d F / d t=0$ (i.e. the nullcline of Eq. (8.b)) is determined by a transcendental equation. This makes it hard to analyze the shape of this curve, how the shape varies with the parameters, and where intersections with the locus $d T / d t=0$ occur. These properties can be investigated numerically. However, a greater insight about how parameter variations cause bistability is obtained by first looking at a reduced model whose behaviour is more elementary to analyze. Although analyzing "a model of the model" may seem frivolous, in fact the process of reducing a model to simple limiting case for initial analysis is a standard technique in applied mathematics. We draw the reader's attention to a classic paper by Ludwig et al. (1978) in which this strategy is used.

\section{3.b Approximating the model by a single equation for trail strength}

Using the definition $S=F / T$, it follows that

$$
\frac{d S}{d t}=-\frac{F}{T^{2}} \frac{d T}{d t}+\frac{1}{T} \frac{d F}{d t}
$$

which, by substitution of the equations for $d T / d t$ and $d F / d t$ results in

$$
\frac{d S}{d t}=-C S^{2}+S-E^{\prime} S e^{-B^{\prime} S}+D
$$

where $C$ and $D$ are:

$$
C=\left(\frac{1}{F}-1+A^{\prime}\right), \quad D=\alpha(1-F) .
$$

Even though the quantities $C$ and $D$ are not actually constants, $C$ can be roughly approximated by $A^{\prime}$, and $D$ is some small positive quantity when the population is mostly followers.

Equation (11) is easily seen to have bistable behaviour. We can locate the positions and determine the number of steady states of this equation (viewed as an equation in $S$ with slowly varying coefficients) by investigating intersections of curves of the form $Y=D+S-C S^{2}$ (a parabola opening down) and $Y=E^{\prime} S e^{-B^{\prime} S}$. (A curve with a single hump and a long tail), as shown in Fig. 2. It is possible to have one, two, or three intersections. Three intersections occur provided that (a) the hump of the exponential curve is higher than the peak of the parabola (e.g. if $E^{\prime}$ is large) (b) the decay of the exponential is rapid so that its curve dips below the parabola and intersects it again twice to the right of the hump (e.g. if $B^{\prime}$ is large). When three intersections do occur, as shown in Fig. 2(a), one finds that the two extremes (low $S$ and high $S$ ) are stable, while the middle value is not. Thus the system will evolve to either strong or weak trails, depending on the initial value of $S$.

One advantage of first understanding eq. (11) is that we can thereby classify the bistability of the trail-following model as one representative of a broader category of bistable models. The population of budworm in the paper of Ludwig, Jones and 
1

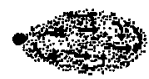

2

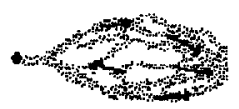

3

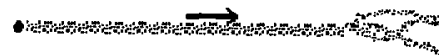

4

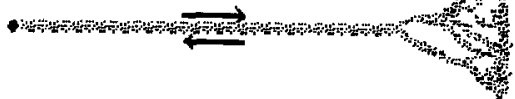

$\mathbf{5}$

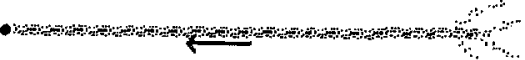

Fig. 1. Stages in formation of a swarm include (a) random exploratory motion, (b) formation of a heavy-traffic trunk trail and a fan-shaped area of exploration. The black dot represents a nest. The model in this paper suggests that variations in the density of the traffic, or in the type of trails can result from small changes in the parameters that characterize the behaviour of individuals. After Raignier and van Boven (1955)
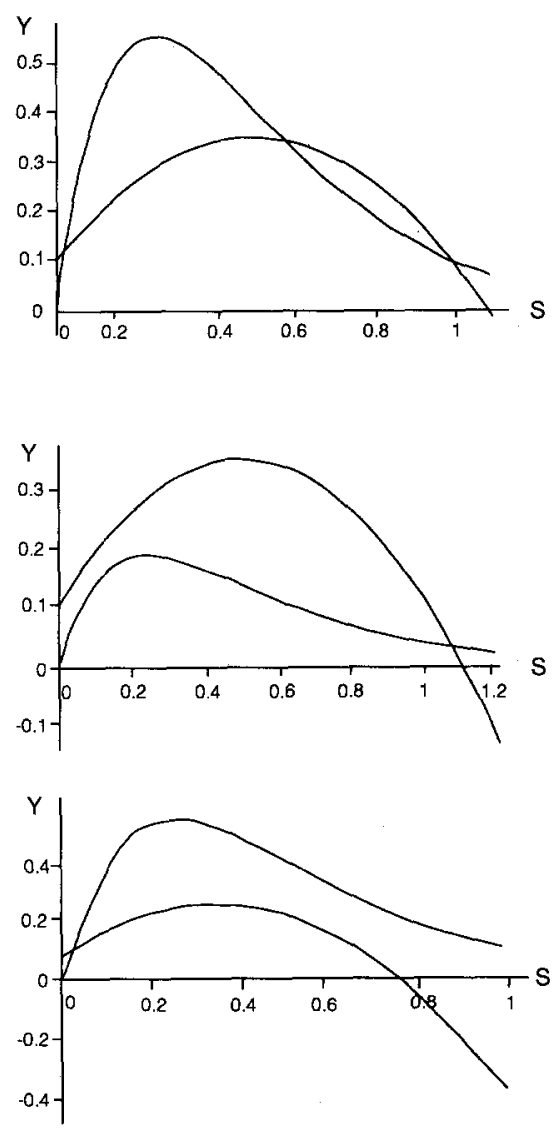

Fig. 2. The behaviour of the reduced model for trail-strength (11) can be understood in terms of the intersections of the two curves shown here. When the parabola intersects the humped curve three times (a), there will be three steady states. The two extreme states (low and high) represent stable weak and strong trails. Other possible configurations in which only strong (b) or only weak (c) trails are formed

Holling (1978) has a similar bistable behaviour (due to intersection of a straight line with a humped curve as above). 


\section{3.c Analysis of the full model equations}

Returning to the full model eqs. $(8 . a, b)$ we note that nullclines are

$$
\begin{array}{ll}
\frac{d T}{d t}=0: & 1-\left(1-A^{\prime}\right) F-T=0, \\
\frac{d F}{d t}=0: & E^{\prime} \frac{F}{T} e^{-B^{\prime} \frac{F}{T}}=\alpha^{\prime}(1-F) .
\end{array}
$$

Thus the nullcline $d T / d t=0$ given by Eq. (12.a) is a straight line with slope $-\left(1-A^{\prime}\right)$ and $T$ axis intercept $T=1$. Since $A^{\prime}=a / v<1$ the slope of this line is negative. The nullcline $d F / d t=0$ given by Eq. (12.b) is transcendental, as previously mentioned. We note that when $F$ approaches 1 , the left hand side of Eq. (12.b) approaches 0 , and so must the right hand side. This can happen only if $T$ approaches 0 (so that $S=F / T$ becomes large, forcing $E^{\prime} \exp \left(-B^{\prime} S\right)$ to zero.) Thus, $T$ approaches zero as $F$ approaches 1 . Other details of this shape are best handled by graphing techniques. For example, the software PhasePlane (G.B. Ermentrout) was used to generate a sequence of nullcline configurations as the parameters $E^{\prime}$, and $B^{\prime}$ are varied. Changing $\alpha^{\prime}$ has the same effect as changing $1 / E^{\prime}$, simply affecting the relative sizes of the linear and exponential terms. The effects of changing $E^{\prime}$ and $B^{\prime}$ are shown in Fig. 3. The curve has an $s$-shape, with one smooth hump, and the possibility of a sharp "knee" close to the origin as the value of $E^{\prime}$ increases (Fig. 3(a)) or equivalently as the value of $\alpha^{\prime}$ decreases. $B^{\prime}$ causes the height of the hump to increase or decrease,as shown in Fig. 4(b), without greatly changing the other features of the curve.

These variations in the shape of the $d F / d t=0$ nullcline influence the number of intersections and the locations of intersections that are made with the straight line $d T / d t=0$. It is possible to obtain one, two, or three intersections. Three extreme possibilities are shown in Fig. 4: In Fig. 4(a) there are three intersections, and thus three steady states of the system (8.a, b). In Figs 4(b), and 4(c), there is a single intersection, but its position differs from one case to the other. The case of two intersections is of marginal interest here, occurring as a fleeting transition between the other cases. (See Appendix 2 for bifurcation diagram).

The phase plane behaviour of these cases (analyzed with PhasePlane) is shown in Fig. 5, corresponding to the three nullcline configurations shown in Fig. 4. The evolution of the length of trails $T$, on the horizontal axis, and the number of followers, $F$, on the vertical axis is given for a number of possible starting values. The final values of $F$ and $T$ depend on the initial values in case (a), but in cases (b) and (c), all initial values lead to the same final result. The analysis of stability properties of the steady states is given in the appendix to this paper.

\section{3.d Interpretation of the phase plane diagrams}

To interpret the meaning of the phase plane diagrams recall that the ratio $S=F / T$ is low near the $T$ axis (where $F$ is small) and high close to the $F$ axis (where $T$ is small). Thus, on the $T F$ planes shown in Figs. 4 and 5, each steady state represent a whole network of trails whose average strength $S$ depends on the position of the steady state. Points close to the $T$ axis (i.e. having low values of $F$ ) represent weak networks, whose degree of coordinated following is low. For example, Fig. 

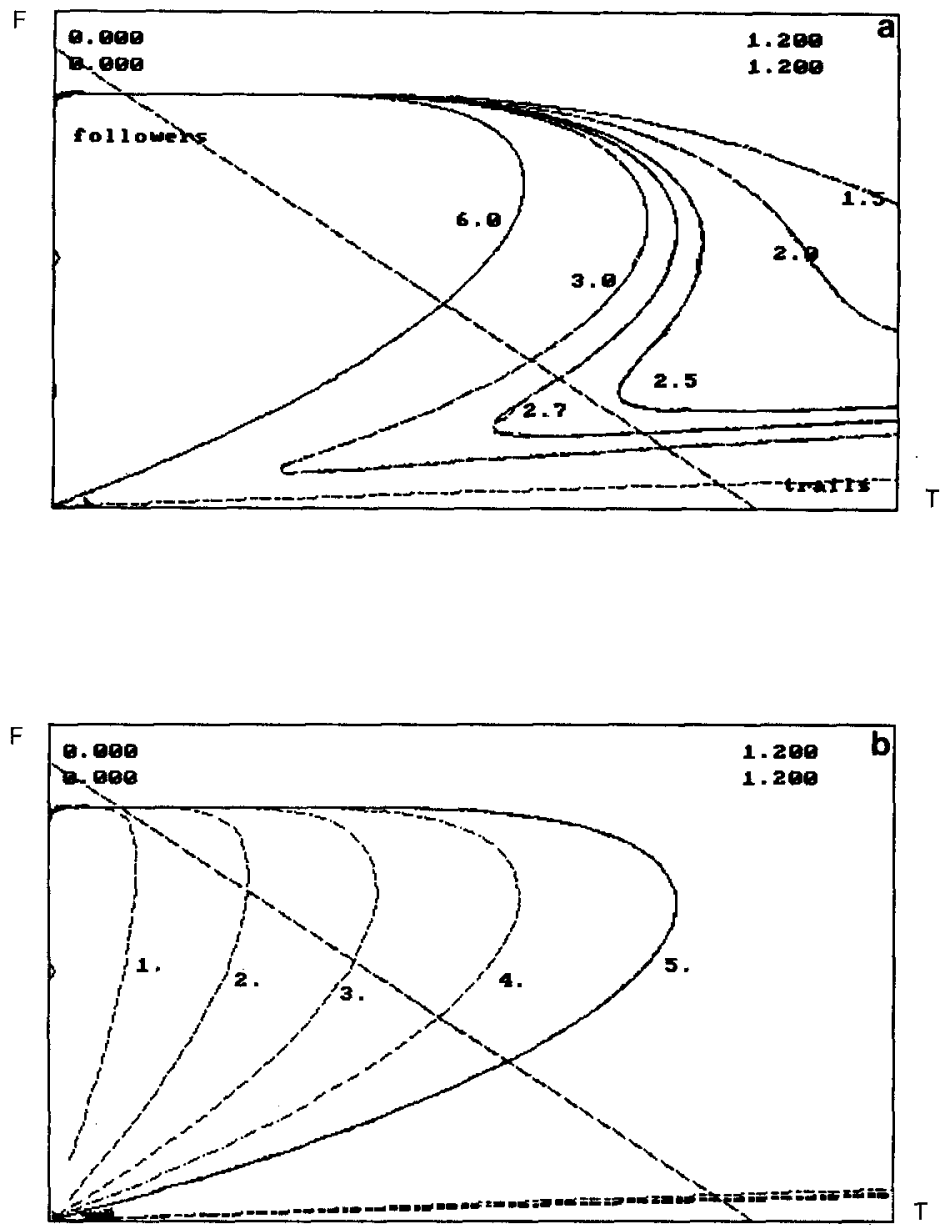

$\mathrm{T}$

Fig. 3. The nullcline $d F / d t=0$ (12.b), whose shape resembles the numeral "2" changes as the parameters $E^{\prime}$ and $B^{\prime}$ are changed. In a, as $E^{\prime}$ increases, the curve gets a hump and a kink, forming up to 3 interesections with the straight nullcline $d T / d t=0$. In $\mathbf{b}$, increasing $B^{\prime}$ enlarges the hump portion of the curve, thus also influencing the number of intersections with the $d T / d t=0$ nullcline. Note for certain values, e.g. near $E^{\prime}=2.6$ in $(a)$ and near $B^{\prime}=1$ in $(b)$, small parameter changes lead to dramatic differences, i.e. bifurcations. Plots generated with PhasePlane (G. B. Ermentrout) with parameter values $A^{\prime}=0.1, \alpha^{\prime}=0.3$. In (a) $B^{\prime}=4.0$. In $(b) E^{\prime}=6.0$

5(b) shows evolution towards a steady state representing a "weak" trail network, on which relatively few individuals are followers. Points close to the $F$ axis (having low values of $T$ ) represent coordinated movement, i.e. a heavy traffic "strong" trails network. (An example is Fig. 5(c)). In Fig. 5(a), there are two stable steady states, one for weak and one for strong trails. Each of the stable steady states has a certain basin of attraction (i.e. set of $(F, T)$ values which are drawn to it), and the sizes and shapes of these basins depends on the parameters in the equations. 

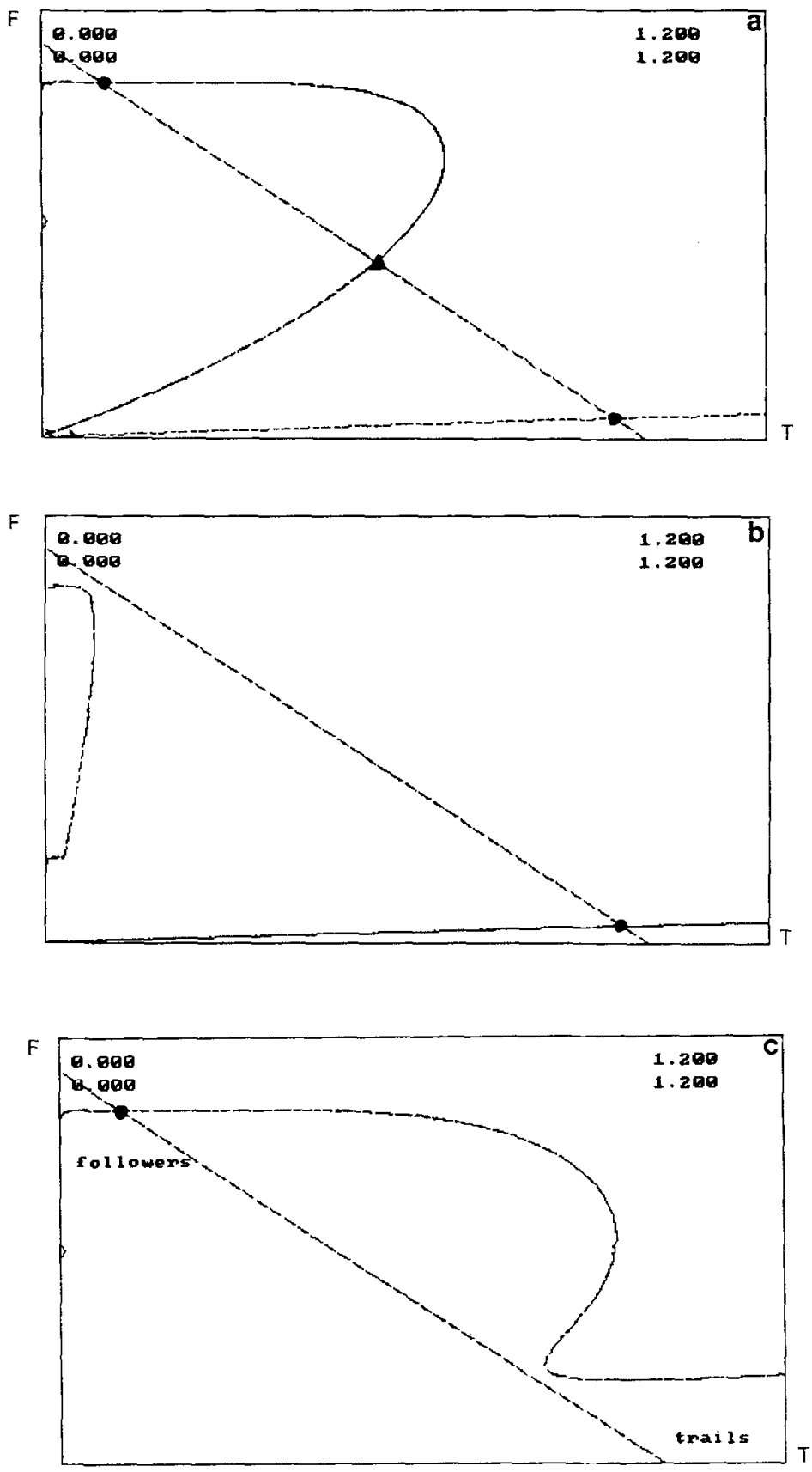

Fig. 4. Three possible configurations of the nullcline $d F / d t=0$ showing how it could interesect the nulleline $d T / d t=0(12, \mathrm{a}, \mathrm{b})$ so as to produce a three steady states, or only one steady state (in $\mathbf{b}$ and $\mathrm{c}$. The strength of trails is proportional to the ratio of $F / T$ (follower traffic density along the trails). Therefore in $(b)$ the steady state represents weak trails. In (c) the steady state represents strong trails. Produced with PhasePlane (G. B. Ermentrout) with $A^{\prime}=0.1, \alpha^{\prime}=0.3$. In (a) $B^{\prime}=4.0$ and $E^{\prime}=6.0$. In $(b) B^{\prime}=0.7$ and $E^{\prime}=6.0$, In $(c) B^{\prime}=4.0$ and $E^{\prime}=2.5$ 

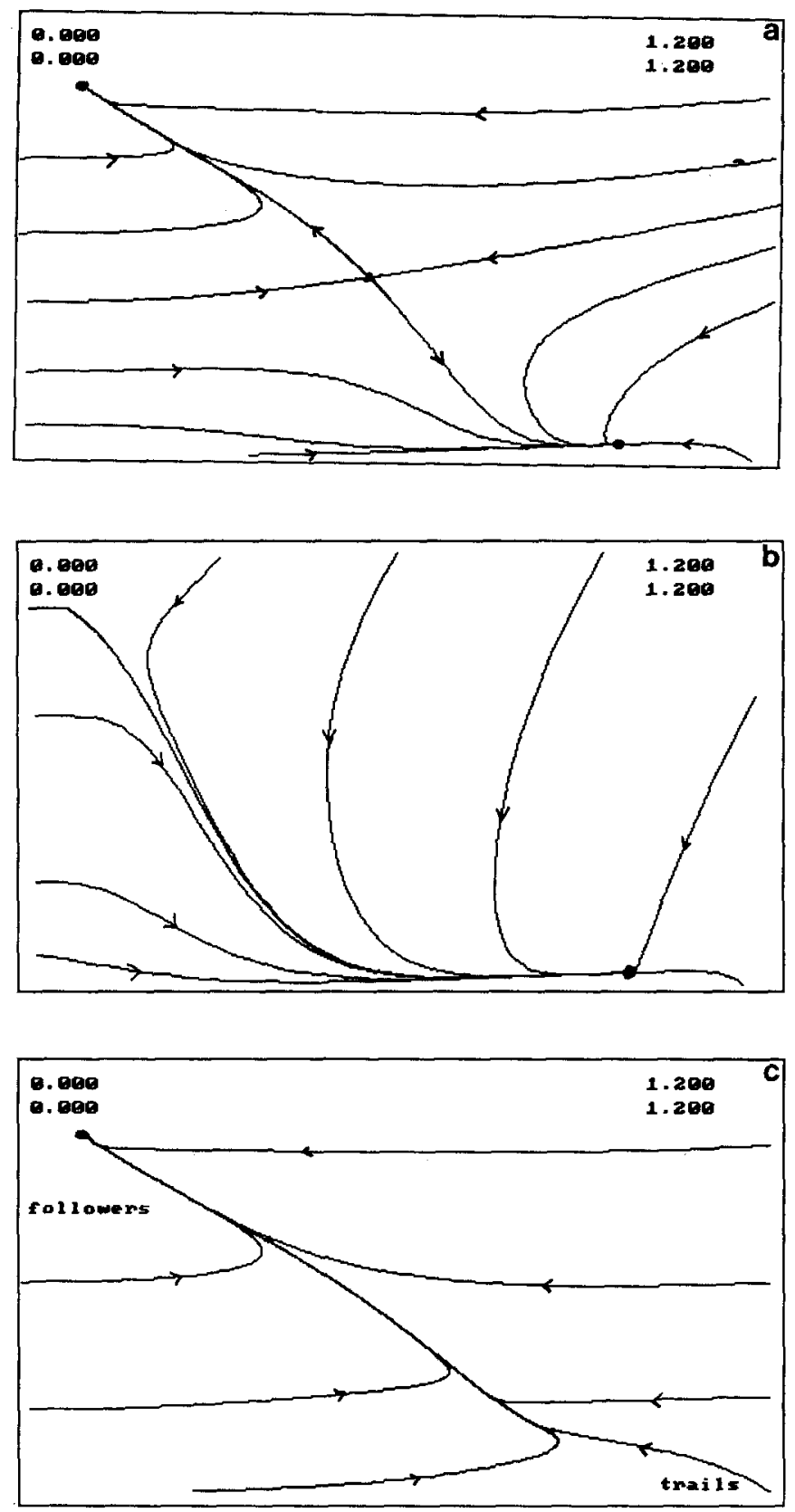

Fig. 5. Phase plane plots of $(12 . a, b)$ corresponding to the cases shown in Figs. $4 a, b$, c. In a there is bi-stable behaviour, in which the strength of the trails can either build up to a stable high level, or decrease to a stable weak level. In cases $\mathbf{b}$ and $\mathbf{c}$ only one of the two trail types is possible. Changes in the tendency of individuals to stray off trails, and in the sensitivity to pheromone concentration can lead to transitions between the above three situations. Plots produced by PhasePlane, with parameters as in Fig. 4 
Even though only the proportion of followers is plotted here, we interpret each curve on these phase plane diagrams as a time course of trail-following since $L=1-F$ (in dimensionless variables) is known once $F$ is known. Taking Fig. 5(b), we see that under all starting conditions, followers decline. As they stray off the trails, a greater percentage of the population becomes exploratory or "lost". Eventually only a small proportion of the population are following trails, and the trails are quite long, and hence weak. In case 5(c), the opposite occurs: exploratory ants that encounter the trails are able to follow them faithfully and increase their strength. Eventually all explorers are absorbed and the trails fill with traffic, becoming strong trails. In case 5(a), the outcome depends on the initial distribution of explorers, followers, and trails. Where the trails are very long, or where the density of followers is too low to keep up the trail strength, a weak network will result. But where there are followers and shorter trails, a strong "trunk trail" will be formed.

Examples of trunk trails that eventually lead to a region of exploration can be seen in the branching trails of army ants drawn by Rettenmeyer (1963). Typically, the first or second branch points in the network may lead to a highly branched region with a fine lace-like appearance of minute tributaries. Because each bifurcation in the network can reduce the traffic density on the branches to $50 \%$ of the parent trail, the border between attraction to the "strong" steady state and the "weak" steady state (e.g. in case of Fig. 6) may be crossed suddenly. This would explain why the transition between trunk trails and exploratory region may be fairly sharp, rather than a gradual bifurcation of increasing order.

Under distinct parameter values, the model leads to one of the three possible qualitative outcomes shown in Fig. 5. As parameter values change, transitions will take place between one case and another. How these transitions occur is of particular relevance to the question of adaptation of the population to its environment. We discuss the findings and the implications in the next section.

\section{3.e How the population adapts to meet its needs}

The model makes several predictions about the plasticity of population behaviour. To understand transitions that might occur we consider the effects of varying the parameters in the model.

A typical example is illustrated by the transitions shown in Fig. 5. The transition from case (a) to (c) is accomplished by varying $E^{\prime}$, the maximal rate of straying off a trail. From Fig. 3(a) one sees that a relatively small change in $E^{\prime}$, from $E^{\prime}=2.5$ to $E^{\prime}=2.7$ brings about a sharp change in nullcline configuration: for $E^{\prime}=2.5$ the nullclines intersect only once (close to $F=1$ ), but for $E^{\prime}=2.7$ two additional intersections exist. The change from 2.5 to 2.7 leads to a transition from exclusively strong trails to trails that can be either strong or weak. The same transition occurs if the parameter $\alpha^{\prime}$ is varied (in the opposite direction).

The transition from Fig. 5(a) to (b) occurs by changing $B^{\prime}$, the degree of sensitivity to trail strength. From Fig. 3(b) we see that when $B^{\prime}<1.0$, the nullclines intersect only once, close to $T=1$. Thus, low values of $B^{\prime}$ correspond to a state in which only weak networks can be formed. As $B^{\prime}$ increases slightly, the hump grows, and two new intersections are formed. Thus, a relatively small change in $B^{\prime}$ gives rise to a switch between strong and weak networks. 
L. Edelstein-Keshet
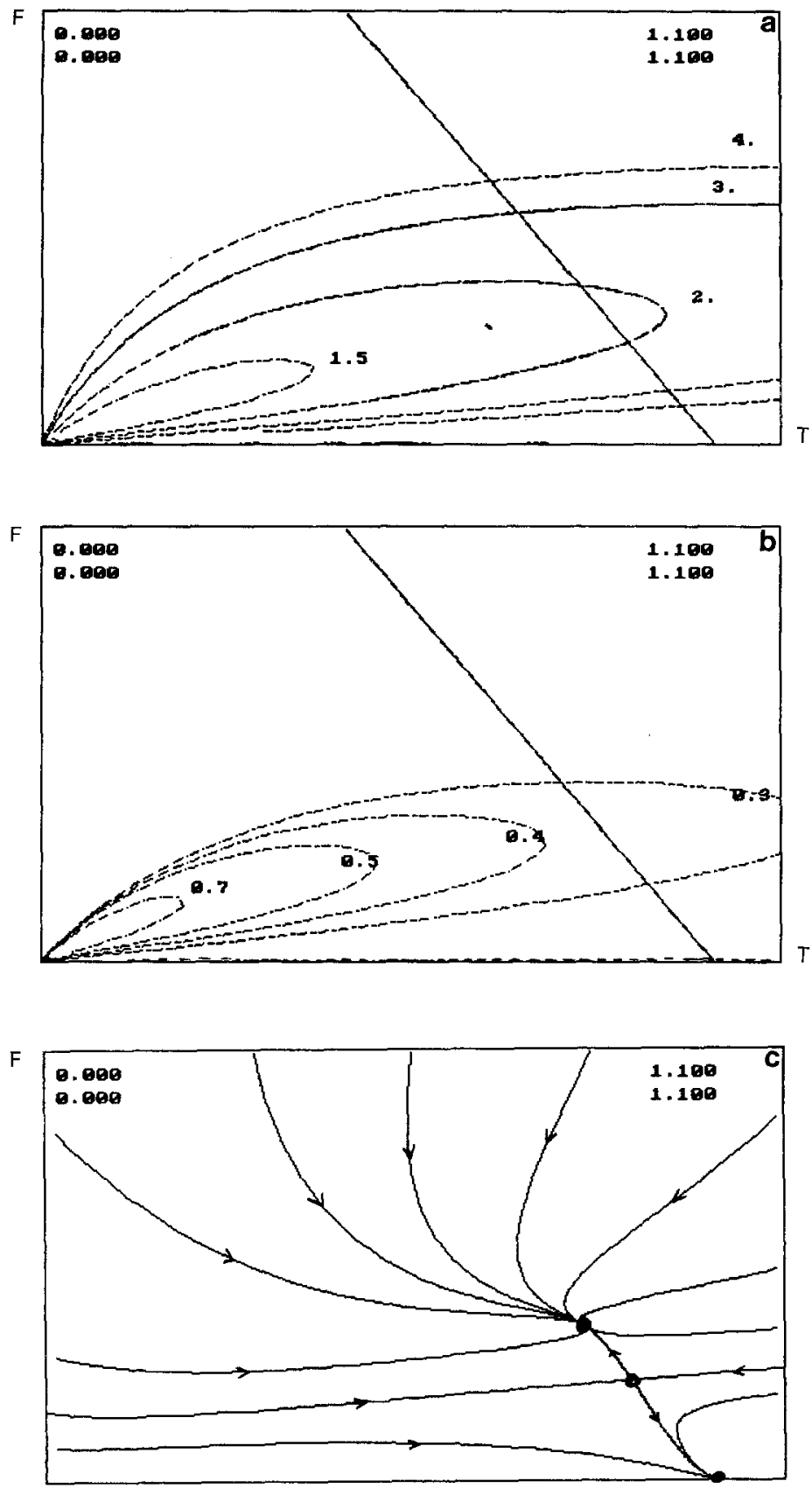

Fig. 6. Behaviour of an alternate model in which attraction to the trails, rather than tendency to lose trails depends on trail strength. Equations $(14 \mathrm{a}, \mathrm{b})$ with parameter values $A^{\prime}=0.5$, were simulated. The nullcline of Eq. (14.b) includes the horizontal $T$ axis and the loop-like curve. a Variations in the value of $\alpha^{\prime}$ cause two steady states to appear when $\alpha^{\prime}$ approaches 2.0. $(k=0.35) \mathbf{b}$ Variations in $k$ have a similar effect $\left(\alpha^{\prime}=2.0\right)$ c phase plane behaviour with $\alpha^{\prime}=2.0$ and $k=0.35$. Three steady states occur. The stable ones represent all weak trails (on the $T$ axis) and a network with some followers (in the 1st quadrant) 
Since $E^{\prime}=E / \Gamma$, and $E$ is the rate of losing a trail, any change in the individual that causes it to lose the trail more frequently, (for example an increased tendency for making random turns) would cause a transition to weak trails. $B^{\prime}=b \Gamma / v$ is proportional to $b$ which represents the increase in the fidelity of followers per unit increase in trail strength. This parameter could be linked to the degree of responsiveness of the pheromone receptors on the antennae of an ant, since, as we have argued (see appendix) increase in $S$ can be associated with an increase in pheromone on trails. Alternately, $b$ could represent the responsiveness of an ant to recruitment via antennal contact with sisters who are following trails. Under conditions of high recruitability ( $b$ large), the population will form strong trails.

Dependence on total population size, $N$ appears only in the dimensionless quantity $\alpha^{\prime}=\alpha v N / \Gamma^{2}$. We have already noted that increasing $\alpha^{\prime}$ is equivalent to decreasing $E^{\prime}$, an effect that promotes strong trails to be formed. This is to be expected since in a large population opportunities for contacts between individuals are more numerous, so that signals for recruitment can be reinforced to a greater extent. It is well-recognized in many theories for self-organizing systems that increasing the size of the population will result in an increase in self-organizing properties of the society (e.g., see papers by Deneubourg).

As previously noted, for a given set of conditions, the fraction of the population on trails will attain a balance with the fraction of explorers. However, changes in conditions will bring about changes in this balance. It is reasonable to expect that environmental influences can affect the values of parameters discussed above. For example, the presence of a centralized food source might increase the rate of antennal signalling, recruiting ants to a single trail leading to the food. On the other hand, the presence of diffuse scattered resources might lead to an increase in the random turning rate of individuals, causing them to fall off trunk routes, and establish exploratory "weak" networks. We can thus understand differences between species of ants, or between a given species operating under different conditions in the context of this model: the same basic rules may apply, but under different conditions the population behaviour will differ.

We can also understand the changes in a single population that might take place over the course of a day or a season. The model predicts that gradual variations in the parameters over time can lead to dramatic transitions in behaviour. This means that a tendency to form a strong or a weak trail network may change suddenly as parameter threshold values are crossed. Thus, though the environment may change gradually, for example at sunset when light and temperature values decline, the population may undergo abrupt changes in behaviour. Following the terminology of dynamical systems it is convenient to refer to such transitions as "behavioural bifurcations".

The above comments suggest one further deduction, albeit a speculative one, about the evolution of trail-following in social organisms. If a colony profits by being able to make transitions in its behaviour rapidly, or in a highly sensitive way, (for example, in response to immediate threats to its existence) it stands to reason that a selective advantage would be gained by operating close to the parameter thresholds that we have characterized above. Thus, one would speculate that evolution would drive societies of trail-followers to gradually gain the characteristics that place them close to behavioral bifurcations. 


\section{3.f Trail strength modulates attraction to trails}

We have so far considered only one possibility, namely that the rate of trail loss drops as the strength of the trail increases. Another possibility is that the attraction of exploratory ants to the trails is a function of trail strength. In Figure 6 we investigate this possibility, and assume that the attraction of exploratory ants to trails increases sigmoidally with the strength of the trail, i.e.

$$
\alpha(S)=\alpha^{\prime} \frac{S^{2}}{k+S^{2}} .
$$

The maximal attraction to trails is $\alpha^{\prime}$ and $k$ is a constant governing the steepness of the response to increasing trail strength. A sigmoidal response depicts a cooperative effect, similar to one encountered in chemical kinetics; the "binding" of one individual to the trail enhances the probability that others will bind. If we use the above dependence on trail strength in place of the previous assumption, we get equations of the form

$$
\begin{aligned}
& \frac{d T}{d t}=(1-F)+A^{\prime} F-T, \\
& \frac{d F}{d t}=-E^{\prime} F+\alpha^{\prime} \frac{\left(\frac{F}{T}\right)^{2}}{k+\left(\frac{F}{T}\right)^{2}}(1-F) T .
\end{aligned}
$$

Similar analysis can be carried out on Eqs. (14.a, b), as shown in Fig. 6(a), (b), and (c). As before, it is found that the number of steady states, the positions of these states, and thus the trail-forming properties of the population will depend on the range of the parameters. Figure 6(a), (b) show configurations of the nullclines of Eqs. (14.a) and (14.b) and the way that the $d F / d t=0$ nullcline changes as the parameters $\alpha^{\prime}$ and $k$ vary. It is found that for low values of $\alpha^{\prime}$ or high values of $k$, only one steady state occurs at $F=0, T=1$, i.e. representing a population in which all individuals are randomly exploring and all trails are simple trails behind these solitary explorers. When $\alpha^{\prime}$ is larger, or when $k$ is small, the possibility of forming stronger trails also exists, as shown in Fig. 6(c).

This example suggests that a variety of assumptions about how individuals respond to trail strength can lead to bistable behaviour, or to the ability to switch between weak and strong trails. The variations lead to slightly different versions of this response. For example, in the case of variable attraction to the trails, formation of strong trails alone does not occur. The reason is that followers always have some fixed probability of losing the trails: the population can never be composed entirely of followers.

\section{3.g Other assumptions about sensitivity to trail strength}

Two different assumptions about how individuals respond to trail strength have been explored in Sect. 2.d and 3.f. We have incorporated each of these assumptions into the basic trail-following model in order to explore the consequences of each one individually. In principle, some combination of these responses may be present.

It is also conceivable that other parameters that have been assumed constant might vary depending on strength of trails. For example, the reinforcement of the 
trails by followers $a$, or the walking speed $v$ may depend on pheromonal concentration (as reported, for example by Deneubourg et al.). If individuals secrete a variable mixture of trail pheromones, it is even conceivable that $\Gamma$, the trail decay rate changes, as the mixture of trail marker changes, and this too, could depend on the trail strength.

It is possible to consider a model in which many possible combinations of such assumptions are tested. Some of these models have more exotic behaviours, with a possibility of multiple steady states, indicative of many possible stable trail networks of varying strengths. It is not the aim of this paper to deal exhaustively with such cases, but merely to document the possible importance of simple responses to trail strength. Rather than consider in detail a long list of possible combinations, it would seem appropriate to pick a single species of ant, determine experimentally the sensitivity of parameters to recruitment signals (such as pheromonal concentration, or number of antennal contacts with other ants) and then further modify and explore the model based on the biological findings.

\section{Discussion}

Although this paper focuses on the example of trail-following in ants, the adaptation of chemical communication for the purpose of coordinating group movement is widespread in the animal kingdom. In the social insects, trail markers are used in exploring and charting routes in a new territory, as well as for mass migration from one nest site to another. (Able 1980, Hölldobler and Wilson 1990). Trail-following also occurs in social bacteria such as Myxobacteria, in larvae and caterpillars (Howard and Flinn 1990, Roessingh 1990, Deneubourg et al. 1990, Fabré 1979), and molluscs (Focardi and Santini 1990, Focardi et al. 1985, Tankersley 1990, Wells and Buckley 1972, Chelazzi et al. 1990). Some mammals, particularly ungulates, have scent glands on their lower legs and feet. Photographs of elk, caribou, wildebeest, and many other migratory or territorial mammals will often reveal a distinct set of trails along which migration takes place. (Able 1980, Estes 1991). The variety of species and conditions in which trail-following occurs suggests that this social behaviour has evolved under the pressure of natural selection in circumstances where group cohesion or coordinated group movement is desirable. It is reasonable to believe that ability to adapt to the environment should thus be "hardwired" into the components of the system. The model in this paper suggests how the responses of individuals to each other, or to chemical markers can result in adaptable group behaviour.

To place the model into the context of previous theoretical work, we review some of the recent literature. Among the first to explore the notion of olfactory (chemical) communication in ants was Wilson (1962a, b). The seminal paper by Bossert and Wilson (1963) laid a foundation for careful analysis of the properties of scent trails, by combining experimental work on the chemical marker of the ant Solenopsis saevissima with a model for diffusion and evaporation of the trail. They thus characterized the length and width of the "active space" surrounding the track of the ant. Sadly, this beautiful paper has not been followed up with investigations of other markers or other species of ants.

Particularly notable is the Brussels groups of investigators (Deneubourg, Pasteels, Goss, Aron, and Calenbuhr) who have pioneered the notion of collective behaviour and self-organization in ants. They have studied trail-following in a combined theo- 
retical, experimental, and numerical treatment. In their papers (Pasteels et al. 1987a, 1987b, 1987c; Goss, et al. 1989, Aron et al. in Alt 1990) locations of nests or food sites and bridges connecting them have been studied. It is shown experimentally that the traffic pattern changes, selecting some bridges or routes at the expense of others. Models for the number of ants and the level of pheromone on each bridge are formulated and analyzed. It is found that usually one route or one bridge is favoured.

Some of the assumptions of these Brussels models are similar to those used in the basic model in this paper. However, the goals of the models differ. I focus on global patterns of behaviour and on the plasticity of this behaviour under variations of parameters. These aspects have not been fully explored in the previous literature.

Simulations of spatial patterns and swarming behaviour appear in Deneubourg et al. (1989, 1990), Franks and Bossert (1989) (Army Ant raiding swarms) and Aron et al. (1990). In some of these simulations the deposition and evaporation of pheromone influences the local direction and speed of motion of the ants. A detailed discussion of the role of parameters in determining the properties of the networks, the branching patterns, or the speed of propagation has not been given in this literature. Watmough (1992) has addressed these questions by analyzing a continuum model for swarming and by simulating the patterns of behaviour using a cellular automata model of ants emerging from a nest, depositing and following trails.

Another point worth noting is the absence in this paper of explicit locations or distribution of food sources. It has been suggested recently by Franks et al. (1992) that differences in food distribution could account for differences in the appearance of trail-networks of a variety of ant species. The model presented here does not contradict this assertion, but rather supports it by revealing how parameters associated with the response of individuals to the environment (including food distribution) can influence the pattern of trails. One could speculate that when food is densely distributed, ants tend to decrease their affinity for trunk trails, for example, by increasing the rate of random turning as they encounter particles of food. This would then cause a transition to a diffuse network of trails suitable for covering a broad area and exploiting the available food. This hypothesis, or variants thereof are experimentally testable.

We have already remarked that all the parameters appearing in the model could, in principle, depend on the environment, the species, and the immediate needs of the colony. (This includes $\alpha$ the rate of attraction to a trail, $\varepsilon$ the rate of losing a trail, $b$ the sensitivity to pheromone concentration, $v$, the velocity of trail-layers, $\Gamma$ the rate of decay of pheromone, and $N$ the total size of the population participating in the activity.)

The model in this paper is among the simplest descriptions for the de novo evolution of a network of trails (in the absence of bridges or other constraints) in which only temporal, and no spatial variation is considered. It suggests that there are several different ways in which a sensitivity to the strength of the trail-marker or to the traffic density on trails would imply an ability to switch between trail-networks suited for different needs. Two examples, in which the ability to follow a trail or the attraction to a trail are strength-dependent have here been studied in detail. The basic equations of the model $(5 . a, b)$ are amenable to testing a variety of other reasonable assumptions, and could serve as a tool for teasing apart several competing hypotheses. An attractive feature of the model is that it is based on experimentally 
measurable parameter values pertaining to individual behaviour. A drawback of this model is that it can only give some global view of the extent to which trails have become organized, without predicting any details about the geometry, spatial extent, or interconnections of the trails. To this end, we have studied a spatial variant of the model and performed simulations showing actual evolutions of trail-patterns. These topics are dealt with in companion papers.

\section{Appendix 1 Relation of trail strength, trail length, and pheromone level}

The trail length equation (2) can be derived by considering pheromonal deposition and evaporation, under a set of simplifying assumptions set forth below. Assume that individuals secrete droplets of fixed concentration pheromone at the rates:

$\phi=$ number of droplets deposited by a follower per unit time,

$\mu=$ number of droplets deposited by a solitary ant per unit time .

Further define

$$
\begin{aligned}
& C(t)=\text { concentration of pheromone per unit area (= \#drops/area), } \\
& \Sigma(t)=\text { amount pheromone per unit length trail (= \#drops/length), } \\
& \Theta(t)=C(t) / \Sigma(t)=\text { average length of trail per unit area. }
\end{aligned}
$$

(Remark: $\Theta(t)$ is the length of trails in the given unit region that would be formed if the droplets are strung out in a linear arrangement with an average distribution of $\Sigma$ droplets per unit length.) A balance for $\mathrm{C}(t)$ would be

$$
d C / d t=[\text { secretion rate }]-[\text { evaporation rate }] \text {. }
$$

We now make the following simplifying assumptions: (1) The walking speeds and rates of secretion of droplets by ants are constant. (2) The pheromone evaporates into the environment which is assumed to be an infinite sink (whose pheromonal concentration $C_{0}$ will be assumed to be zero) (3) Evaporation is equivalent to decay in the number of droplets per unit area at a rate proportional to $\left\{C(t)-C_{0}\right\}$ (i.e. for modelling evaporation, $C(t)$ represents a chemical concentration).

Then the equation for $C(t)$ is

$$
\frac{d C}{d t}=\mu L+\Phi F-\gamma\left(C-C_{0}\right) \text {. }
$$

Where $F(t)$ and $L(t)$ are followers and solitary ants per unit area as before. The reason for formulating the equation in terms of $C(t)$ is that the term for evaporation makes most sense in terms of a concentration difference, and the meaning of $C(t)$ is closest to that of a true concentration. Now setting $C_{0}=0$ and substituting

$$
C(t)=\Theta(t) \Sigma(t)
$$

$t$ into the above equation, one obtains

$$
\frac{d(\Sigma \Theta)}{d t}=\Sigma \frac{d \Theta}{d t}+\Theta \frac{d E}{d t}=\mu L+\phi F-\gamma \Sigma \Theta
$$

This equation demonstrates that deposition of pheromone can cause trails to elongate (increase $\Theta$ ) or to attain a higher net pheromonal level per unit length (increase $\Sigma$ ) or both. We now consider the following limiting cases: 
(1) $\Sigma=S_{0}=$ constant; (The solitary ants are depositing pheromone on unmarked ground, and the followers are replacing droplets that have evaporated from trails, but not significantly increasing the number of droplets on a given length of trail). Then letting $T(t)=\Theta(t)$, substituting into (A1) and dividing by $S_{0}$ yields:

$$
\begin{aligned}
& \frac{d T}{d t}=\left(\frac{\mu}{S_{0}}\right) L+\left(\frac{\phi}{S_{0}}\right) F-\gamma T, \\
& \frac{d T}{d t}=v L+a F-\gamma T
\end{aligned}
$$

where we have defined

$$
\nu=\frac{\mu}{S_{0}} \quad a=\frac{\phi}{S_{0}} .
$$

Under this limiting case, we obtain the trail-length Eq. (2).

(2) $\Theta=T_{0}=$ constant; (The ants are mostly walking over old trails and reinforcing the pheromonal marker, and there are few explorers $L \approx 0$ ). Then letting $S(t)=\Sigma(t)$, substituting $\Theta=T_{0}$ into (A.2), setting $L=0$ and dividing by $T_{0}$ yields

$$
\begin{aligned}
& \frac{d S}{d t}=\left(\frac{\mu}{T_{0}}\right) L+\left(\frac{\phi}{T_{0}}\right) F-\gamma S, \\
& \frac{d S}{d t}=\Phi F-\gamma S .
\end{aligned}
$$

Here $\left(\Phi=\phi / T_{0}\right)$. In this limiting case the strength of the trails (in the meaning used in the model) coincides with the chemical level of pheromone along the trails) since at equilibrium (when $d S / d t=0$ ), $S(t)$ would be proportional to $F / T$. (As mentioned previously, the ratio of $F / T$ represents a true pheromonal strength only in this limiting case. In general it is an average taken over all trails, including weak ones, and is consequently lower than the chemical strength of any one given strong trail).

\section{Appendix 2 Analysis of model eqs. (8.a, b)}

We first investigate the number and stability properties of steady states to Eqs. (8.a, b). Typically, there are three steady states, with the middle one an unstable saddle point, as we shall show below. However, bifurcations leading to the disappearance of the saddle point with one of the other steady states occur as a parameter such as $B^{\prime}$ is varied.

To discuss stability in the case of three equilibria, we define the function

$$
H(T, F)=-E^{\prime} F e^{-B^{\prime} \frac{F}{T}}+\alpha^{\prime}(1-F) T .
$$

Then the nullcline of Eq. (8.b) is the curve $H(T, F)=0$ in the $T F$ plane. By implicit differentiation, it follows that the slope of this curve is

$$
m=\left.\frac{d F}{d T}\right|_{H=0}=-\frac{H_{T}}{H_{F}} .
$$




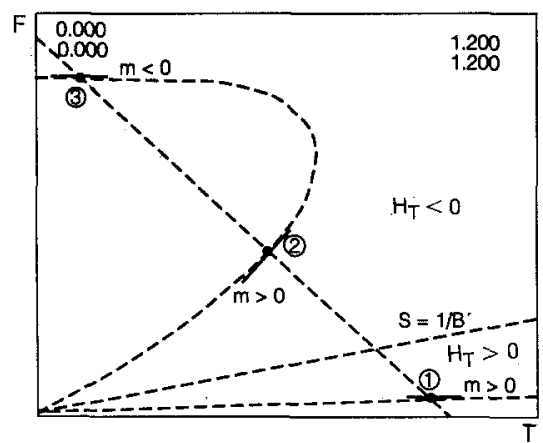

Stability of the steady states of Eqs. $(8 . a, b)$ are determined from the Jacobian,

$$
J=\left[\begin{array}{cc}
-1 & A^{\prime}-1 \\
H_{T} & -\frac{H_{T}}{m}
\end{array}\right]
$$

where subscripts indicate partial differentiation with respect to the variable $T$, and where we have set $H_{F}=-H_{T} / m$ in the fourth entry. We now observe that

$$
\operatorname{Trace}(J)=-1-\frac{H_{T}}{m}, \quad \operatorname{Det}(J)=H_{T}\left(\frac{1}{m}+1-A^{\prime}\right) .
$$

By assumptions in the model, the quantity $1-A^{\prime}$ is always positive, since $A^{\prime}<1$. Stability thus depends on the sign of $H_{T}$ and the sign and magnitude of $m$. An elementary calculation reveals that at steady state,

$$
\begin{aligned}
& H_{T}=\frac{1}{T}\left[-B^{\prime} S\left(E^{\prime} F e^{-B^{\prime} S}\right)+\alpha^{\prime}(1-F) T\right], \\
& H_{T}=\alpha^{\prime}(1-F)\left(1-B^{\prime} S\right) .
\end{aligned}
$$

Since $F \leqq 1$, the sign of $H_{T}$ is positive for $S<1 / B^{\prime}$ and negative for $S>1 / B^{\prime}$. This splits the $F T$ plane into two triangular regions, separated by the line $S=$ $F / T=\left(1 / B^{\prime}\right)$. (See Fig. 7) Above the line, $H_{T}$ is negative, and below the line it is positive. We consider a configuration shown in Fig. 7. The table below establishes the sign pattern of the quantities $H_{T}$ and the nullcline slope $m$ for each of the three intersections (steady states 1,2 , and 3 ) It can be seen from the effective signs of the determinant and the Jacobian that the middle intersection is a saddle point, while the two extreme ones are stable nodes or spirals. (Remarks: in steady state $3, m$ is a small negative number since $F$ cannot increase above $F=1$. The sign of Trace $(J)$ is not applicable (NA) in case (2) since the negative determinant always results in saddle point behaviour).

The basins of attraction of the stable steady states can be found by plotting the stable manifold of the saddle point. A typical example is shown in Fig. 8. The grey region is the basin of attraction of the weak trail steady state (at the lower right corner). The white region is the basin of attraction of the strong trail steady state, at the upper left.

The bifurcation diagram for Eqs. (8.a, b) shown in Fig. 9 was produced by Gerda de Vries using the simulation program AUTO. Here $B^{\prime}$ is the bifurcation parameter. The number and stability properties of the steady states can be seen as a function of the value of $B^{\prime}$. It is evident that two saddle-node bifurcations occur, one for $B^{\prime}$ increasing from 0 , and for $B^{\prime}$ decreasing from 5 (See figure legends for exact values of the parameters used.) 
Table 1. Stability properties of the steady states

\begin{tabular}{llllll}
\hline State & $H_{T}$ & $m$ & $\operatorname{Trace}(J)$ & $\operatorname{Det}(J)$ & Stability \\
\hline$(1)$ & + & + & - & + & Stable \\
$(2)$ & - & + & NA & - & Saddle \\
$(3)$ & - & - & - & + & Stable \\
\hline
\end{tabular}

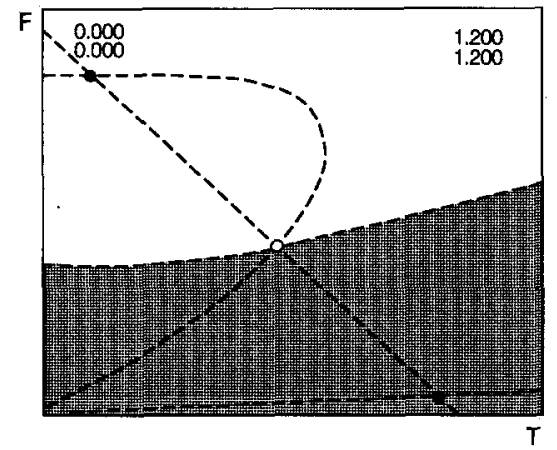

Fig. 8. The unstable manifold of the saddle point subdivides the $T F$ plane into basins of attraction of the two equilibria. Starting values below this separatrix (shaded) will evolve to the lower steady state, whereas those above the separatrix will be attracted by the upper steady state

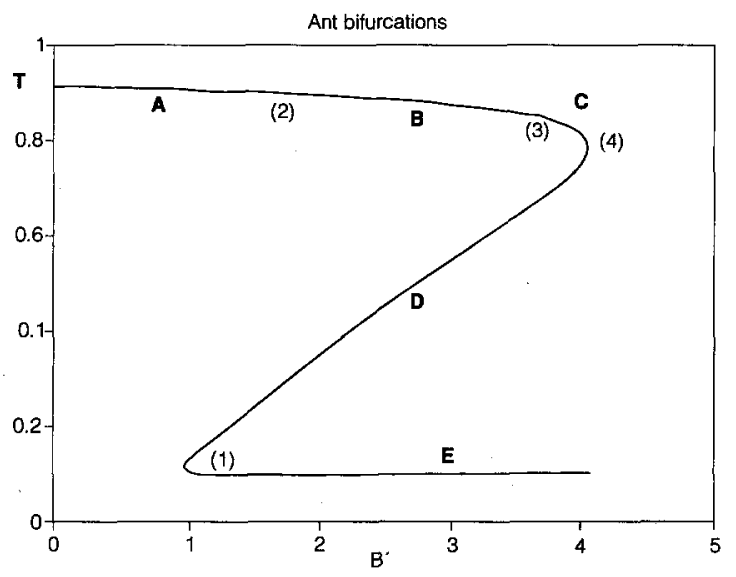

Fig. 9. Bifurcation diagram of $(8 \mathrm{a}, \mathrm{b})$ produced by Gerda de Vries using the simulation package AUTO. The equations were simulated using the following values of the parameters: $A^{\prime}=0.1$, $E^{\prime}=2.6, \alpha^{\prime}=0.3$. The bifurcation parameter was $B^{\prime}$. The regions marked

along this curve indicate $(A)$ two real negative eigenvalues (stable node), $(B)$ complex eigenvalues with negative real parts (stable spiral), $(C)$ two real, negative eigenvalues, $(D)$ two real eigenvalues of opposite signs (saddle point) $(E)$ Two real negative eigenvalues. The values of $B^{\prime}$ at the transition points are: (1) 0.9817 (2) 1.798 , (3) 3.746 , (4) 4.0838 . The vertical axis is the steady state(s) value of $T$

Acknowledgement. LEK gratefully acknowledges support from the National Sciences and Engineering Research Council of Canada under grant number OGPIN 021. I wish to thank J. Watmough and B. Ermentrout for helpful comments and discussion of this model. I am particularly indebted to Gerda de Vries for her help in running AUTO, and for her time spent producing Fig. 9.

\section{References}

Able, K. P. Mechanisms of Orientation, Navigation, and Homing. In: Gauthreaux, S. A. (ed.) Animal Migration, Orientation, and Navigation, pp. 283-373. New York: Academic Press 1980

Alt, W., Hoffmann, G. (eds.) Biological Motion. Proceedings, Königswinter 1989. (Lect. Notes Biomath., vol 89) Berlin, Heidelberg New York, Springer 1990

Aron, S., Deneubourg, J. L., Goss, S., Pasteels, J. M.: Functional Self-organization illustrated by inter-nest traffic in ants: the case of the argentine ant. In: Alt, W., Hoffmann, G. (eds.) Biological 
Motion. Proceedings, Königswinter 1989 (Lect. Notes Biomath., vol. 89, pp. 533-547) Berlin Heidelberg New York: Springer 1990

Aron, S., Pasteels, J. M., Goss, S., Deneubourg, J. L.: Self-organizing spatial patterns in the Argentine ant Iridomyrmex humilis (Mayr). In: Vander Meer, R. K., Jaffe, K., Cedeno, A. (eds.) Applied Myrmecology, A World Perspective, pp. 438-451. Boulder, CO: Westview

Aron, S., Pasteels. J. M., Denoubourg, J. L.: Trail-laying behaviour during exploratory recruitment in the Argentine ant, Iridomyrmex humilis (Mayr). Biol. Behav. 14(3), 207-217 (1989)

Beckers, R., Deneubourg, J. L., Goss, S., Pasteels, J. M.: Collective decision making through food recruitment. Insectes Soc. 37(3), 258-267 (1990)

Bossert, W. H., Wilson, E. O.: The analysis of olfactory communication among animals. J. Theor. Biol. 5, 443-469 (1963)

Calenbuhr, V., Deneubourg, J. L.: A model for trail following in ants: individual and collective behaviour. In: Alt, W., Hoffmann, G. (eds.) Biological Motion. Proceedings, Königswinter 1989. (Lect. Notes Biomath., vol. 89, pp 453-469) Berlin Heidelberg New York: Springer 1990

Camazine, S.: Self-organizing pattern formation on the combs of honey-bee colonies. Behav. Ecol. Sociobiol. 28, 61-76 (1991)

Chelazzi, G., Della Santina, P., Parpagnoli, D.: The role of trail following in the homing of intertidal chitons: A comparison between three Acanthopleura spp. Mar. Biol, 105(3), 445-450 (1990)

Deneubourg, J. L., Aron, S., Goss, S., Pasteels, J. M.: The self-organizing exploratory pattern of the Argentine ant. J. Insect Behav. 3(2), 159-168 (1990)

Deneubourg, J. L., Goss, S., Franks, N., Pasteels, J. M.: The blind leading the blind: chemically mediated morphogenesis and army ant raid patterns. J. Insect Behav. 2, 719-725 (1989)

Deneubourg, J. L., Goss, S.: Collective patterns and decision making, Ethology. Ecol. Evol. 1, 295-311 (1990)

Deneubourg, J. L., Grégoire, J. C., Le Fort, E.: Kinetics of larval gregarious behavior in the bark beetle Dendeoctonus micans Coleoptera: Scolytidae. J. Insect. Behav. 3, 169-182 (1990)

Edelstein-Keshet, L., Mathematical Models in Biology, New York: Random House 1988

Edelstein-Keshet, L., Ermentrout, G. B.: Models for branching networks in two dimensions. SIAM J. Appl. Math. 49(4), 1136-1157 (1989)

Edelstein-Keshet, L., Ermentrout, G. B.: Models for contact-mediated pattern formation: cells that form parallel arrays. J. Math. Biol. 29, 33-58 (1990)

Ermentrout, G. B., Edelstein-Keshet, L.: Cellular automata approaches to biological modelling. J. Theor. Biol. 160(1), 97-133 (1993)

Estes, D. E.: The Behavior Guide to African Mammals: Including Hoofed Mammals, Carnivores, Primates Berkeley: University of California Press 1991

Fabré, J. H.: Insectes. New York: Felix Gluck Press, C. Scribners 1979

Focardi, S., Deneubourg, J. L., Chelazzi, G.: How shore morphology and orientation mechanisms can affect the spatial organization of intertidal molluscs. J. Theor. Biol. 112, 771-782 (1985)

Focardi, S., Santini, G.: A simulation model of foraging excursions in intertidal chitons, In: Alt, W., Hoffmann, G. (eds,) Biological Motion. Proceedings, Königswinter 1989 (Lect. Notes Biomath., vol 89, pp. 319-330) Springer Berlin Heidelberg New York: 1990

Franks, N. R.: Army ants: a collective intelligence. Am. Sci 77, 139-145 (1989)

Franks, N. R., Bossert, W. H.: The influence of swarm raiding army ants on the patchiness and diversity of a tropical leaf litter ant community. In: Sutton, S. L., Whitmore, T. C., Chadwick, A. C. (eds.) Tropical Rainforest: Ecology and Management, pp. 151-163. Oxford: Blackwell 1983

Franks, N. R., Gomez, N., Goss, S., Deneubourg, J. L.: The blind leading the blind in Army Ant raid patterns: testing a model of self-organization (Hymenoptera: Formicidae). J. Insect. Behav. 4, 583-607 (1991)

Goss, S., Aron, S., Deneubourg, J. L., Pasteels, J. M.: Self-organized shortcuts in the Argentine ant. Naturwissenschaften 76, 579-581 (1989)

Hölldobler, B., Wilson, E. O.: The Ants. Cambridge, MA: Harvard University Press 1990

Howard, R. W., Flinn, P. W.: Larval trails of Cryptolestes ferrugineus (Coleoptera: Cucujidae) as kairomonal host-finding cues for the parasitoid Cephalonomia waterstoni (Hymenoptera: Bethylidae). Ann. Entomol. Soc. Am. 83(2) 239-245 (1990)

Ludwig, D., Jones, D. D., Holling, C. S.: Qualitative analysis of insect outbreak systems: the spruce budworm and forest. J. Anim. Ecol. 47, 315-332 (1978) 
Pasteels, J. M., Deneubourg, J. L. (eds.) From Individual to Collective Behaviour in Social Insects. Basel: Birkhäuser 1987

Pasteels, J. M., Deneubourg, J. L., Goss S.: Transmission and amplification of information in a changing environment: the case of insect societies. In: Prigogine, I., Sanglier, M. (eds.) Law of Nature and Human Contact, pp. 129-156. Bruxelles: G.O.R.D.E.S. 1987a

Pasteels, J. M., Deneubourg, J. L., Goss, S.: Self-organization mechanisms in ant societies. (I) Trail recruitment to newly discovered food sources. In: Pasteels, J. M., Deneubourg, J. L. (eds.) From Individual to Collective Behavior, pp. 155-175. Basel: Birkhäuser 1987b

Raignier, A., van Boven, J.: Étude taxonomique, biologique et biométrique des Dorylus du sousgenre Anomma (Hymenoptera: Formicidae). Ann. Museé R. Congo Belge, IV, Sec. 2, 1-359 (1955)

Rettenmeyer, C. W.: Behavioral studies of army ants. Univ. Kansas Sci. Bull. 44(9), 281-465 (1963)

Roessingh, P.: Chemical marker from silk of Yponomeuta cagnagellus J. Chem. Ecol. 16(7), 2203$2216(1990)$

Schneirla, T. C.: Army Ants. In: (Topoff, H. R. (ed.) A Study in Social Organization. San Francisco: Freeman 1971

Tankersley, R. A.: Trail following in Littorina littorata: The influence of visual stimuli and the possible role of tracking in orientation. VELIGER 33(1), 116-123 (1990)

Watmough, J.: Models for swarming behaviour in Army Ants. Msc Thesis. Vancouver, BC: University of British Columbia 1992

Wells, M. J., Buckley, S. K. L. Snails and Trails. Anim. Behav. 20, 345-355 (1972) 